\title{
The Mg I line: a new probe of the atmospheres of evaporating exoplanets (Research Note)
}

\author{
V. Bourrier ${ }^{1,2,3}$, A. Lecavelier des Etangs ${ }^{1,2}$, and A. Vidal-Madjar ${ }^{1,2}$ \\ ${ }^{1}$ CNRS, UMR 7095, Institut d'astrophysique de Paris, 98bis boulevard Arago, 75014 Paris, France \\ e-mail: bourrier@iap.fr \\ 2 UPMC Univ. Paris 6, UMR 7095, Institut d'Astrophysique de Paris, 98bis boulevard Arago, 75014 Paris, France \\ 3 Observatoire de l'Université de Genève, 51 chemin des Maillettes, 1290 Sauverny, Switzerland
}

Received 17 December 2013 / Accepted 22 October 2014

\begin{abstract}
Transit observations of HD 209458b in the UV revealed signatures of neutral magnesium escaping the planet's upper atmosphere. The absorption detected in the Mg I line provides unprecedented information on the physical conditions at the altitude where the atmospheric blow-off takes place. Here we use a 3D model of atmospheric escape to estimate the transit absorption signatures in the $\mathrm{Mg}$ I line of their host stars. The detectability of these signatures depends on the brightness of the star and the escape rate of neutral magnesium. We identify a sample of potentially evaporating exoplanets that covers a wide range of stellar and planetary properties, and whose extended exospheres might be detected through Mg I line observations with current UV facilities, allowing further steps in comparative exoplanetology.
\end{abstract}

Key words. surveys - planets and satellites: atmospheres - methods: numerical

\section{Introduction}

Planetary atmospheres outside our solar system have been characterized using observations of transiting planets. In particular, Lyman- $\alpha$ observations of atomic hydrogen in the atmospheres of HD 209458b and HD 189733b revealed that hot Jupiters evaporate, significant amounts of gas escaping their gaseous atmosphere (e.g., Vidal-Madjar et al. 2003; Lecavelier des Etangs et al. 2010). Heavier elements were identified at high altitudes in the extended exosphere of HD 209458b, in the lines of O I, C II, and Si III (Vidal-Madjar et al. 2004; Linsky et al. 2010; Ben-Jaffel \& Sona Hosseini 2010), Si IV (Schlawin et al. 2010), and Mg I (Vidal-Madjar et al. 2013). Signatures of escaping particles have also been found in the Lyman- $\alpha$ line of the warm Jupiter $55 \mathrm{Cnc}$ b (Ehrenreich et al. 2012) and the hot-Neptune GJ436b (Kulow et al. 2014), and in the Mg II line of the hot Jupiter WASP-12b (Fossati et al. 2010; Haswell et al. 2012). A wide variety of models has been developed to explain these observations, which are well explained by the blow-off of the atmosphere (see Bourrier \& Lecavelier des Etangs 2013 and references therein): the strong X/extreme-UV (EUV) heating from the star heats the whole upper atmosphere to such a degree that it is placed in a hydrodynamic state, in some cases overflowing the Roche lobe (Lecavelier des Etangs et al. 2004). Observations of escaping atmospheres are for now limited to very few cases, and yet estimates of the evaporation state of transiting exoplanets from determinations of their energy budget (e.g., Lecavelier des Etangs 2007; Ehrenreich \& Désert 2011) show that smaller planets may also bear significant mass losses, as shown by the recent result of Kulow et al. (2014) for the hot Neptune GJ 436b. Here we propose to evaluate the evaporation state of known transiting exoplanets and to calculate the dynamics of escaping neutral magnesium atoms in order to estimate their detectability with current facilities.

\section{Atmospheric escape of neutral magnesium from HD 209458b}

Using the echelle E230M grating of the Space Telescope Imaging Spectrograph (STIS) instrument onboard the Hubble Space Telescope (HST), Vidal-Madjar et al. (2013) observed the transit of the hot Jupiter HD 209458b in the lines of neutral (2852.9641 $\AA$ ) and singly ionized magnesium (2796.3518 and $2803.5305 \AA$ ). While no atmospheric absorption was observed in the Mg II line, the authors detected a blue-shifted transit absorption signature in the velocity range -62 to $-19 \mathrm{~km} \mathrm{~s}^{-1}$ in the $\mathrm{Mg}$ I line, with an absorption depth of $8.8 \pm 2.1 \%$. Bourrier et al. (2014) used a 3D particle model of the dynamics of the escaping magnesium atoms, coupled with an analytical modeling of the atmosphere below the exobase, to reproduce these observations. They found that the observations are best reproduced if the exobase is close to the Roche lobe and neutral magnesium particles escape from the exobase at a rate of $2.9_{-0.9}^{+0.5} \times 10^{7} \mathrm{~g} \mathrm{~s}^{-1}$ with a velocity of $25 \mathrm{~km} \mathrm{~s}^{-1}$.

\section{Modeling magnesium escape from transiting exoplanets}

To identify the best targets for observations of magnesium escape, we applied a modified version of the model of Bourrier et al. (2014) to the known transiting exoplanets. We calculated 
the theoretical absorption profile generated by neutral magnesium in their atmosphere to derive the significance level of its signature in the $\mathrm{Mg}$ I line.

Bourrier et al. (2014) reported that the electron density needed to reproduce the detected $\mathrm{Mg}$ I signature was surprisingly high. Here, acknowledging possible overestimation of the electron density (possibly due to erroneous radiative and dielectronic recombination rates, higher temperature, or an incorrect estimate of the Mg II absorption), we assumed a collisionless exosphere and used no input on this quantity. The model of Bourrier et al. (2014) was used to constrain the dynamics (and not the ionization) of the magnesium atoms and predict the absorption profile at $2853 \AA$ from scaling the detection in HD 209458b. Hereafter parameters labeled 209 refer to the planet HD 209458b.

Physical properties of exoplanets were extracted from online databases (Wright et al. 2011; Schneider et al. 2011). In September 2013, 142 transiting planets had all the parameters required by the model, in particular to calculate the escape rate of neutral magnesium with the energy budget (e.g., Ehrenreich \& Désert 2011) and to estimate the Mg I line spectrum of each host star.

\section{Estimation of the planetary system properties}

Neutral magnesium atoms escape the atmosphere of a planet at the exobase radius $R_{\text {exo }}$. It was fixed as a best guess by scaling the atmospheric structure of the planet to that of HD 209458b, using the atmospheric scale height $H$, except when the resulting altitude is higher than the Roche lobe,

$R_{\text {exo }}=\min \left(R_{\mathrm{p}}+\frac{H}{H^{209}}\left(R_{\text {exo }}^{209}-R_{\mathrm{p}}^{209}\right), R_{\text {Roche }}\right)$.

Atmospheric mass loss depends on the X/EUV stellar energy flux received by the upper atmosphere. We calculated the potential energy with Eq. (12) in Erkaev et al. (2007), which takes into account the contribution of tidal forces through a correction factor $K_{\text {tide. }}$. The escape rate of neutral magnesium was obtained using the atmospheric mass fraction of this species, $A_{\mathrm{Mg}}$, so that

$\dot{M}_{\mathrm{Mg}}=A_{\mathrm{Mg}} \eta \frac{3 F_{\mathrm{X} / \mathrm{EUV}}(1 \mathrm{AU})}{4 G d_{\mathrm{tr}}^{2} \bar{\rho} K_{\text {tide }}}$,

with $\bar{\rho}$ the mean density of the planet, $F_{\mathrm{X} / \mathrm{EUV}}(1 \mathrm{AU})$ the X/EUV flux per unit area at $1 \mathrm{AU}$ from the star, $d_{\text {tr }}$ the star-to-planet distance at the center of the transit, and $\eta$ the heating efficiency. We assumed that giant gaseous planets with $\bar{\rho}<3 \mathrm{~g} \mathrm{~cm}^{-3}$ have the chemical composition of the Sun $\left(A_{\mathrm{Mg}}=7.08 \times 10^{-4}\right.$; Asplund et al. 2009). For the 34 denser planets in our list of transiting planets we used the mass fraction of neutral magnesium found in the meteorites of the solar system $\left(A_{\mathrm{Mg}}=9.55 \times\right.$ $10^{-2}$ ). For 55 Cnc, HD 209458, HD 189733, and GJ 436 we used the X/EUV stellar emission determined by Sanz-Forcada et al. (2011). For 129 other stars we calculated the stellar EUV flux from its correlation with the rotation velocity of the star (e.g., Wood et al. 1994; Ehrenreich \& Désert 2011). For the 9 remaining stars we estimated the EUV luminosity as a function of the stellar type using Eq. (1) in Lecavelier des Etangs (2007). The heating efficiency $\eta$ is one of the less constrained values of the physics of exoplanet atmospheres (e.g., Lammer et al. 2009; Owen \& Jackson 2012). We calculated escape rates in two limit scenarii, either with $\eta=30 \%$, or with $\eta=100 \%$. We fixed $30 \%$ as a lower limit because it yields a $1 \sigma$ detection in the range -62

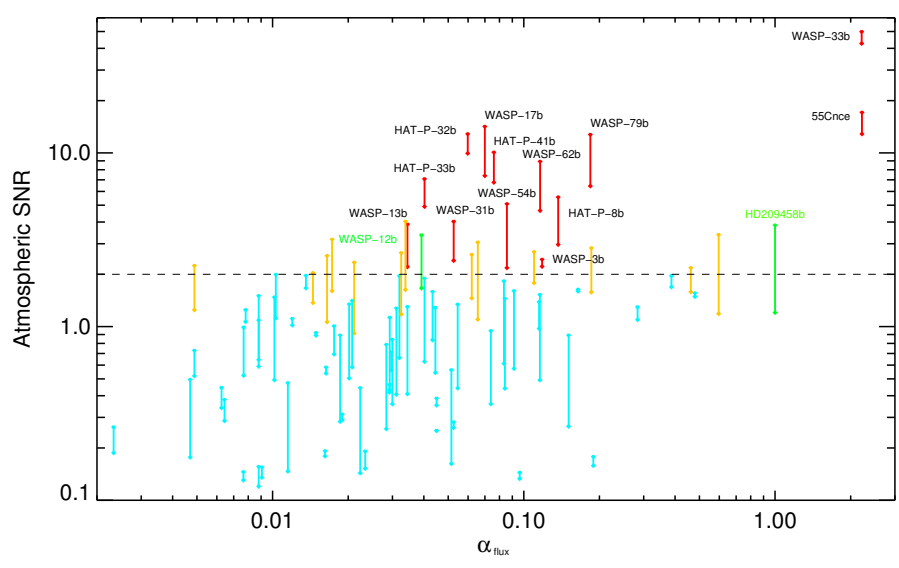

Fig. 1. $\mathrm{S} / \mathrm{N}$ of absorption signatures generated by neutral magnesium in the atmospheres of known transiting exoplanets as a function of the brightness of their host stars in the MgI line seen from Earth. For each planet the lower (upper) $\mathrm{S} / \mathrm{N}$ corresponds to a heating efficiency $\eta=30 \%(100 \%)$. Planets with an $\mathrm{S} / \mathrm{N}$ greater than 2 for $\eta=30 \%$ are plotted in red, and planets with an $\mathrm{S} / \mathrm{N}$ greater than 2 for higher heating efficiencies in orange. Other planets are plotted in blue and the specific hot Jupiters HD 209458 b and WASP-12 b in green.

to $-19 \mathrm{~km} \mathrm{~s}^{-1}$ for HD 209458b, while the absorption signature was detected at $3.6 \sigma$.

For the $\mathrm{Mg}$ I line stellar profiles we used the spectrum of HD 209458b. This spectrum is scaled to a given star with the coefficient $\alpha_{\text {flux }}$, which takes into account the stellar type and the distance from Earth to the star,

$$
\begin{aligned}
\alpha_{\text {flux }} & =\frac{F_{*}^{\mathrm{MgI}}}{F_{209}^{\mathrm{MgI}}} \\
& =10^{-0.4\left(V_{*}-V_{209}\right)-1.2\left((B-V)_{*}-(B-V)_{209}\right)} .
\end{aligned}
$$

This scaling law is derived from measurements taken from the IUE archive. For $55 \mathrm{Cnc}$ we used the IUE spectrum of the G8V star Tau Ceti. For WASP-33 we used the emission spectrum of $\beta$ Pictoris (A6 star), retrieved from the STScI archive.

The dynamics of particles escaping an exoplanet atmosphere is naturally dependent on radiation pressure from the host star. For a magnesium atom the ratio of the radiation force to the stellar gravity $\beta_{*}$ is proportional to the stellar $\mathrm{Mg}$ I line flux received by the escaping atom at its radial velocity $v$,

$\beta_{*}(v)=c^{\mathrm{MgI}} M_{*}^{-1} F_{*}^{\mathrm{Mg} \mathrm{I}}(1 \mathrm{AU}, v)$,

with $c^{\mathrm{Mg} \mathrm{I}}$ a coefficient that only depends on the properties of the $\mathrm{Mg} \mathrm{I}$ line and the atom of magnesium, $M_{*}$ the mass of the star, and $F_{*}^{\mathrm{MgI}}$ the stellar flux at $1 \mathrm{AU}$ from the star at the wavelength corresponding to the Doppler velocity $v$.

The spectral errors on the fluxes were estimated assuming that they are dominated by photon noise (i.e., proportional to the square root of the flux, using the gain of real STIS observations of HD 209458), and then propagated to estimate the uncertainty on the absorption signal.

\section{Detectability of escaping magnesium particles}

Magnesium particles escaping an exoplanet atmosphere are detected through their absorption profile in the $\mathrm{Mg}$ I line of 
Table 1. Properties of the planets of interest and their host stars.

\begin{tabular}{lccccc}
\hline \hline Planet & $\begin{array}{c}M_{\mathrm{p}} \\
\left(M_{\mathrm{jup}}\right)\end{array}$ & $\begin{array}{c}\log \dot{M}_{\mathrm{Mg}} \\
\left(\mathrm{g} \mathrm{s}^{-1}\right)\end{array}$ & $\begin{array}{c}\log \dot{M}_{\mathrm{t}} \\
\left(\mathrm{g} \mathrm{s}^{-1}\right)\end{array}$ & $\begin{array}{c}\log \dot{M}_{\mathrm{t} 3 \sigma} \\
\left(\mathrm{g} \mathrm{s}^{-1}\right)\end{array}$ & $S / N$ \\
\hline WASP-33 b & 2.05 & 10.20 & 13.35 & 11.04 & $42.7 / 49.9$ \\
55 Cnc e & 0.03 & 8.07 & 9.09 & 7.82 & $12.9 / 17.1$ \\
HAT-P-32 b & 0.95 & 10.14 & 13.29 & 12.25 & $9.9 / 12.9$ \\
WASP-17 b & 0.51 & 9.13 & 12.28 & 11.50 & $7.4 / 14.2$ \\
HAT-P-41 b & 0.80 & 9.31 & 12.46 & 11.75 & $6.8 / 10.1$ \\
WASP-79 b & 0.89 & 8.93 & 12.08 & 11.42 & $6.4 / 12.8$ \\
HAT-P-33 b & 0.77 & 9.17 & 12.32 & 11.89 & $4.9 / 7.1$ \\
WASP-62 b & 0.56 & 8.26 & 11.41 & 11.02 & $4.7 / 8.9$ \\
HAT-P-8 b & 1.29 & 8.39 & 11.54 & 11.54 & $3.0 / 5.6$ \\
WASP-31 b & 0.48 & 8.71 & 11.86 & 12.05 & $2.4 / 4.0$ \\
WASP-3 b & 2.01 & 8.45 & 11.60 & 11.87 & $2.2 / 2.4$ \\
WASP-13 b & 0.48 & 8.06 & 11.21 & 11.48 & $2.2 / 3.9$ \\
WASP-54 b & 0.63 & 8.24 & 11.39 & 11.67 & $2.2 / 5.1$ \\
\hline WASP-12 b & 1.36 & 9.10 & 12.25 & 12.74 & $1.7 / 3.4$ \\
HD 209458 b & 0.69 & 7.08 & 10.23 & 11.03 & $1.2 / 3.8$ \\
\hline
\end{tabular}

Notes. $\mathrm{S} / \mathrm{N}$ are given for $\eta=30 \%$ and $100 \%$. Escape rates are calculated for neutral magnesium $\left(\dot{M}_{\mathrm{Mg}}\right)$ and the total material in the atmosphere $\left(\dot{M}_{\mathrm{t}}\right)$ when $\eta=30 \%$, while $\dot{M}_{\mathrm{t} 3 \sigma}$ indicates the total escape rate required to obtain an $\mathrm{S} / \mathrm{N}$ of 3 .

the host star, and we thus studied the signal-to-noise ratio $(\mathrm{S} / \mathrm{N})$ of this absorption signature. In Fig. 1 we show the $\mathrm{S} / \mathrm{N}$ for the 142 exoplanets we studied, as a function of $\alpha_{\text {flux }}$ for heating efficiencies between $30 \%$ and $100 \%$. We caution that the $\mathrm{S} / \mathrm{N}$ were calculated as if the planet transits were observed with HST/STIS in the same conditions as HD 209458b, that is, for a total of about eight hours of observations with the instrument efficiency at $2853 \AA$. Thirteen planets stand out because of the high detectability of their extended atmospheres: $55 \mathrm{Cnc}$ e, HAT-P-8 b, HAT-P-33 b, HAT-P-41 b, WASP-3 b, WASP-13 b, WASP-17 b, WASP-31 b, WASP-33 b, WASP-54 b, WASP-62 b, WASP-79 b, and HAT-P-32 b. Their evaporation state is such that they would still yield an $\mathrm{S} / \mathrm{N}$ between 2 and 40 even with a conservative heating efficiency of $30 \%$ (Table 1 ). We caution that the evaporation of WASP-33 b and HAT-P-32 b is most likely limited by a low heating efficiency becaue with $\eta=100 \%$ these planets would evaporate in $1.6 \mathrm{~Gy}$ and $900 \mathrm{My}$, while they orbit stars aged 10-400 Myr (Collier Cameron et al. 2010; Moya et al. 2011) and 3.8 +0.5 Gy (Hartman et al. 2011). There are thirteen other planets whose evaporating atmosphere would be detectable if they had high heating efficiencies $(S / N>2$ with $\eta=100 \%)$ : HAT-P-6 b, HAT-P-9 b, HAT-P-24 b, HAT-P-40 b, HD 149026 b, HD 209458 b, KELT-2A b, KELT-3 b, Kepler-20 b, TrES-4 b, WASP-1 b, WASP-12 b, WASP-67 b, WASP-78 b, and XO-4 b. One of them is HD 209458b, for which a positive detection of escaping magnesium was obtained, and WASP-12b, for which ionized magnesium was detected at high altitude in the exosphere (Fossati et al. 2010; Haswell et al. 2012). A higher heating efficiency may not necessarily lead to a proportionally higher $\mathrm{S} / \mathrm{N}$ because the absorption depth depends not only on the escape rate, but also on self-shielding effects and the optical thickness of the extended atmosphere. In Fig. 2 we display the $\mathrm{S} / \mathrm{N}$ as a function of the escape rate and the radiation-pressure-induced acceleration of escaping particles, which control the absorption depth and the spectral range of the absorption signature. The combination of high escape rates with high acceleration generates the most significant absorption signatures, which is

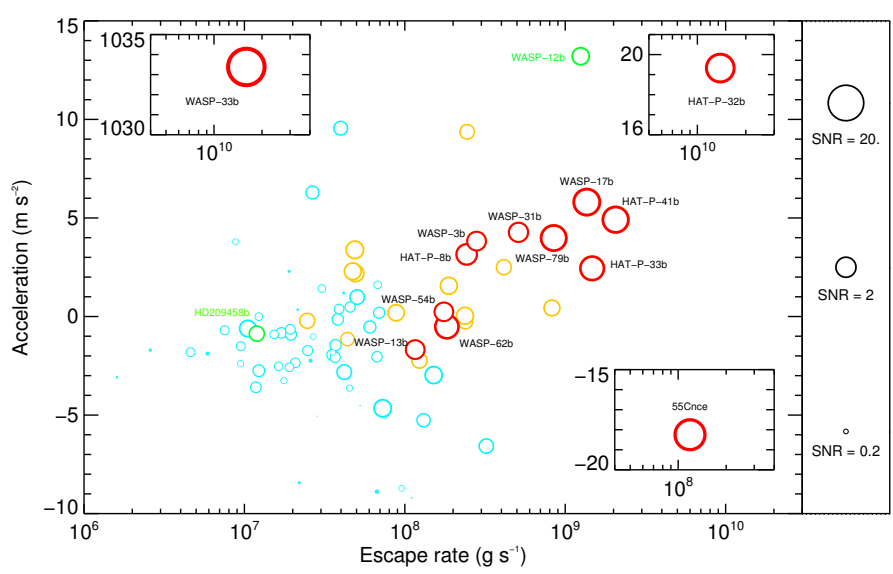

Fig. 2. $\mathrm{S} / \mathrm{N}$ as a function of the escape rate and the radiation-pressureinduced acceleration on escaping particles. Each planet is represented by a disk whose size varies with the S/N (heating efficiency is $30 \%$ ). Colors are the same as in Fig. 1. Most planets with a high $\mathrm{S} / \mathrm{N}$ are found to have high escape rates and high accelerations.

favored by planets orbiting bright stars at short orbital distances. Interestingly, the hot Jupiter HD 189733 b, usually one of the best targets for atmospheric observations (in the Lyman- $\alpha$ line and from the near-UV to the mid-IR), is a poor candidate for observations of magnesium escape. This is mainly due to the low flux in the $\mathrm{Mg}$ I line of its $\mathrm{K} 2 \mathrm{~V}$ star.

\section{Discussion and conclusion}

Escaping atmospheres have been detected in a very limited number of exoplanets, mainly through transit observations of the H I Lyman- $\alpha$ line, and more recently, of the Mg I line. While neutral hydrogen allows characterizing the exosphere far from the planet, magnesium can be used in addition to probe lower altitudes in the transition region between the thermosphere and the exosphere. We used a 3D model of atmospheric escape, initially developed for neutral magnesium escaping HD 209458 b (Vidal-Madjar et al. 2013; Bourrier et al. 2014), to simulate the escape of this heavy species from the atmospheres of known transiting exoplanets and to evaluate the resulting absorption signatures in the $\mathrm{Mg}$ I line. The detectability of such signatures depends on the escape rate of neutral magnesium, the brightness and size of the star, and on the strength of its radiation pressure. We identified a sample of thirteen planets $(55 \mathrm{Cnc}$ e, HAT-P-8 b, HAT-P-33 b, HAT-P-41 b, WASP-3 b, WASP-13 b, WASP-17 b, WASP-31 b, WASP-33 b, WASP-54 b, WASP-62 b, WASP-79 b, and HAT-P-32 b) with high escape rates of ten to a thousand times higher than for HD 209458b, which are expected to produce atmospheric signatures that can be detected with current UV facilities such as the HST (they have an S/N of between about 2 and 50 in the least favorable conditions). Because of the detection of ionized magnesium in its exosphere, WASP-12 b would also be an interesting planet to search for neutral magnesium. This sample covers a wide range of planetary and stellar properties, from the very-hot Earth-like planet $55 \mathrm{Cnc}$ e to the young A-star orbiting planet WASP-33 b. Observations of this sample would allow comparing exoplanet upper atmospheres and provide an unprecedented vision of the blow-off mechanism with constraints on the velocity, temperature, and density structure of the escaping gas in the thermosphere - exosphere transition region (Bourrier et al. 2014). We can also anticipate that 
observations in the UV with the HST/STIS instrument would not only allow detecting neutral and ionized magnesium in their atmospheres, but also very likely the detection of many other heavy species such as Mn I, Mn II, Fe I, or Fe II.

Acknowledgements. We thank the referee, and offer particular thanks to the editor for its support. The authors acknowledge the support of the French Agence Nationale de la Recherche (ANR), under program ANR-12-BS05-0012 ExoAtmos. This work has also been supported by an award from the Fondation Simone et Cino Del Duca. This research has made use of the Extrasolar Planets Encyclopaedia at exoplanet.eu as well as the Exoplanet Orbit Database and the Exoplanet Data Explorer at exoplanets.org.

\section{References}

Asplund, M., Grevesse, N., Sauval, A. J., \& Scott, P. 2009, ARA\&A, 47, 481 Ben-Jaffel, L., \& Sona Hosseini, S. 2010, ApJ, 709, 1284

Bourrier, V., \& Lecavelier des Etangs, A. 2013, A\&A, 557, A124

Bourrier, V., Lecavelier des Etangs, A., \& Vidal-Madjar, A. 2014, A\&A, 565, A105

Collier Cameron, A., Guenther, E., Smalley, B., et al. 2010, MNRAS, 407, 507 Ehrenreich, D., \& Désert, J.-M. 2011, A\&A, 529, A136

Ehrenreich, D., Bourrier, V., Bonfils, X., et al. 2012, A\&A, 547, A18

Erkaev, N. V., Kulikov, Y. N., Lammer, H., et al. 2007, A\&A, 472, 329
Fossati, L., Haswell, C. A., Froning, C. S., et al. 2010, ApJ, 714, L222 Hartman, J. D., Bakos, G. Á., Torres, G., et al. 2011, ApJ, 742, 59 Haswell, C. A., Fossati, L., Ayres, T., et al. 2012, ApJ, 760, 79

Kulow, J. R., France, K., Linsky, J., \& Loyd, R. O. P. 2014, ApJ, 786, 132

Lammer, H., Odert, P., Leitzinger, M., et al. 2009, A\&A, 506, 399

Lecavelier des Etangs, A. 2007, A\&A, 461, 1185

Lecavelier des Etangs, A., Vidal-Madjar, A., McConnell, J. C., \& Hébrard, G. 2004, A\&A, 418, L1

Lecavelier des Etangs, A., Ehrenreich, D., Vidal-Madjar, A., et al. 2010, A\&A, 514, A72

Linsky, J. L., Yang, H., France, K., et al. 2010, ApJ, 717, 1291

Moya, A., Bouy, H., Marchis, F., Vicente, B., \& Barrado, D. 2011, A\&A, 535, A110

Owen, J. E., \& Jackson, A. P. 2012, MNRAS, 425, 2931

Sanz-Forcada, J., Micela, G., Ribas, I., et al. 2011, A\&A, 532, A6

Schlawin, E., Agol, E., Walkowicz, L. M., Covey, K., \& Lloyd, J. P. 2010, ApJ, 722, L75

Schneider, J., Dedieu, C., Le Sidaner, P., Savalle, R., \& Zolotukhin, I. 2011, A\&A, 532, A79

Vidal-Madjar, A., Lecavelier des Etangs, A., Désert, J.-M., et al. 2003, Nature, 422, 143

Vidal-Madjar, A., Désert, J.-M., Lecavelier des Etangs, A., et al. 2004, ApJ, 604, L69

Vidal-Madjar, A., Huitson, C. M., Bourrier, V., et al. 2013, A\&A, 560, A54

Wood, B. E., Brown, A., Linsky, J. L., et al. 1994, ApJS, 93, 287

Wright, J. T., Fakhouri, O., Marcy, G. W., et al. 2011, PASP, 123, 412 